\title{
Liesegang Rings Engineered from Charged Nanoparticles
}

\author{
István Lagzi, Bartlomiej Kowalczyk, and Bartosz A. Grzybowski* \\ Department of Chemical and Biological Engineering and Department of Chemistry, Northwestern University, \\ 2145 Sheridan Road, Evanston, Illinois 60208-3113
}

Received August 13, 2009; E-mail: grzybor@ northwestern.edu

The study of spatial patterns that are formed from reacting and diffusing chemicals has been central to the development of nonlinear chemical kinetics ${ }^{1,2}$ and has provided mechanistic understanding of natural phenomena ranging from the formation of stalactites and striated minerals ${ }^{3}$ through intracellular oscillations ${ }^{4}$ to cardiac rhythms. ${ }^{5,6}$ Although virtually all artificial pattern-forming chemical systems [Liesegang rings (LRs), ${ }^{6,7}$ Belousov-Zhabotinsky (BZ) oscillations and waves, ${ }^{8}$ and discharge filaments ${ }^{9}$ ] have been serendipitously discovered rather than systematically designed, subsequent developments have led to remarkable control of these patterns. Nowadays, BZ waves can be propagated controllably, ${ }^{10}$ Turing patterns can be designed rationally from first principles, ${ }^{11}$ and Liesegang-like structures can be crafted with desired shapes and interband spacings in the presence ${ }^{12}$ or absence ${ }^{13}$ of external fields. In the context of Liesegang structures, however, it must be remembered that they are observed for a relatively few combinations of coprecipitating chemicals and supporting media. ${ }^{2,14}$ While the majority of LR systems are based on ionic species, we wished to investigate whether it might be possible to create such structures from larger charged entities. In particular, systems of functionalized, oppositely charged nanoparticles (NPs) are interesting candidates since (i) their precipitation behavior is governed by the condition of charge neutrality ${ }^{15}$ [which can be adjusted by changing the composition of self-assembled monolayers (SAMs) ${ }^{16}$ stabilizing these particles] and not by the solubility product (which is set for a given pair of salts) and (ii) the NPs can have cores made of different materials, so the forming LRs could have variable compositions, including those that have not been realized in ionic systems. Here we demonstrate that when coupled to NP diffusion, these properties do lead to the formation of distinct Liesegangtype structures made of charged, metallic NPs of various types. The formation of these LRs is rationalized using a theoretical model based on the solubility thresholds deriving from the NP chargeneutrality condition. Our results demonstrate that appropriately engineered nanoscopic components can serve as building blocks for self-organizing chemical patterns.

We used Au or Ag nanoparticles [average metal core diameters in different samples ranging from 7 to $10 \mathrm{~nm}$ with standard deviations $(\sigma)$ of $6-42 \%$ ] stabilized with $\mathrm{SAMs}^{16}$ of either positively charged $N, N, N$-trimethyl(11-mercaptoundecyl)ammonium chloride (TMA NPs, from ProChimia Poland) or negatively charged mercaptoundecanoic acid (MUA NPs; fully deprotonated at $\mathrm{pH} 11$ ) or mixed SAMs of one of these thiols and uncharged 11-mercapto1-undecanol (MUO) (Figure 1a). In a typical experiment (Figure $1 \mathrm{~b})$, a $2 \mathrm{~mm}$ thick sheet of $0.5 \mathrm{wt} \%$ agarose gel was soaked in a solution of NPs of one polarity (the "inner electrolyte"; concentration 1.13-4.52 $\mathrm{mM}$ in terms of metal atoms in the NPs). A circular reservoir of radius $r_{0}=7.5 \mathrm{~mm}$ was then cut in the gel and filled with a solution of oppositely charged NPs (the "outer electrolyte", 7.4-31.3 mM). As the outer electrolyte diffused into the gel and the inner electrolyte migrated toward the reservoir, these species a)

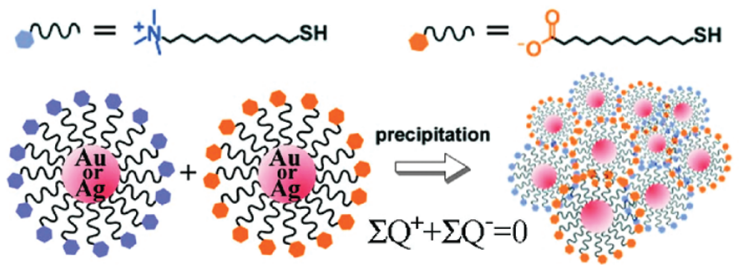

b) $t_{0}=0$

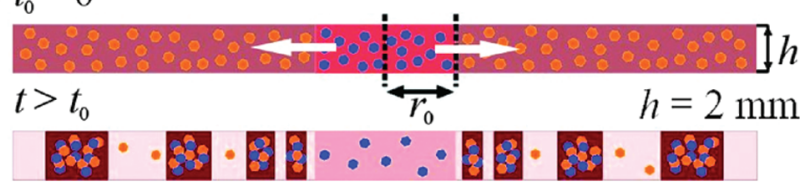

c)

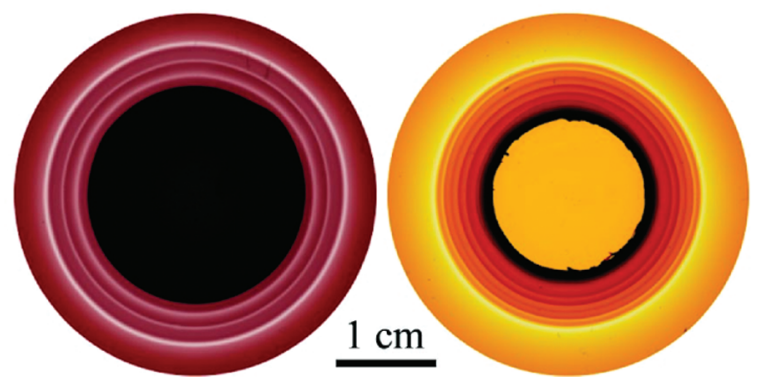

Figure 1. Liesegang rings made of oppositely charged nanoparticles. (a) Structures of the charged alkane thiols $\mathrm{HS}-\left(\mathrm{CH}_{2}\right)_{11}-\mathrm{N}\left(\mathrm{CH}_{3}\right)^{3+}$ (TMA) and $\mathrm{HS}-\left(\mathrm{CH}_{2}\right)_{10}-\mathrm{COO}^{-}$(MUA) used to coat the NPs. Irrespective of the properties of the metal cores, oppositely charged NPs precipitate when their charges are balanced. (b) Cross-sectional view and typical dimensions of the gel layer supporting formation of NP-based LR patterns. The rings appear after time $t \approx 24-36 \mathrm{~h}$. (c) Examples of ring structures formed by (left) $7.5 \mathrm{~nm}$ AuTMA (10.43 mM) NPs delivered to a gel containing $7.4 \mathrm{~nm}$ AuMUA (2.26 mM) NPs and (right) $9.7 \mathrm{~nm}$ AgTMA (9.47 mM) NPs delivered into a gel soaked with $7.4 \mathrm{~nm}$ AgMUA (2.08 mM) NPs. The characteristic hues of the patterns derive from the surface plasmon resonances (SPRs) of the particles (red-violet for Au NPs and yellow-orange for Ag NPs).

aggregated and formed distinct bands/rings. Figure 1c shows examples of such rings made of AuTMA/AuMUA (left picture) and AgTMA/AgMUA (right picture) NPs. We note that no rings were observed when (i) NPs of one or both types were uncharged or (ii) when the NPs were larger than $12-15 \mathrm{~nm}$, in which case spatially continuous NP aggregation was mostly due to van der Waals rather than electrostatic forces. ${ }^{15,17}$

For a given type and concentration of the charged NPs, the LRs obey the spacing law of consecutive ring radii: ${ }^{2,18} r_{n+1} / r_{n}=p$, where $p$ is the so-called spacing coefficient (Figure 2a). Also, when the NP concentrations change, the value of $p$ decreases with increasing concentration of either the inner or outer electrolyte (Figure $2 b-d$ ). This behavior is reminiscent of the so-called Matalon-Packter law ${ }^{13}$ for ionic systems, which relates $p$ to the initial concentrations of the inner $\left(c_{0}^{\text {in }}\right)$ and outer $\left(c_{0}^{\text {out }}\right)$ electrolytes: $p\left(c_{0}^{\text {in }}, c_{0}^{\text {out }}\right)=F\left(c_{0}^{\text {in }}\right)+$ $G\left(c_{0}^{\text {in }}\right) / c_{0}^{\text {out }}$, where $F\left(c_{0}^{\text {in }}\right)$ and $G\left(c_{0}^{\text {in }}\right)$ are decreasing functions of $c_{0}^{\text {in }}$. 


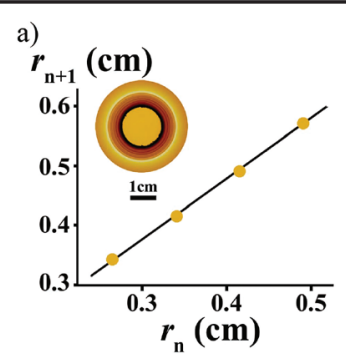

b)

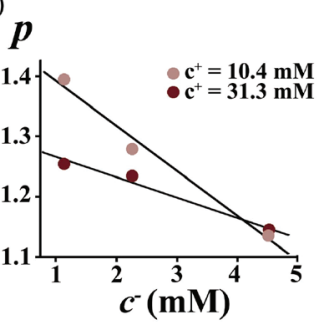

c)

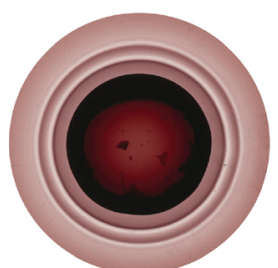

d)
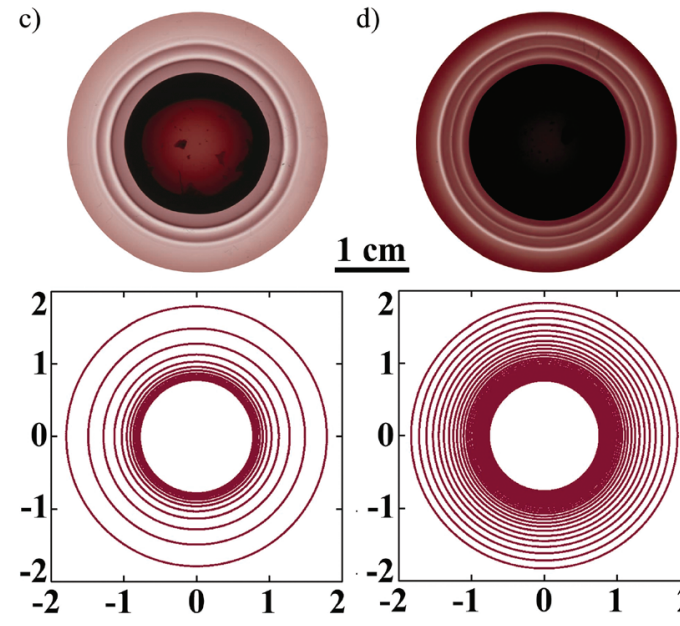

$1 \mathrm{~cm}$

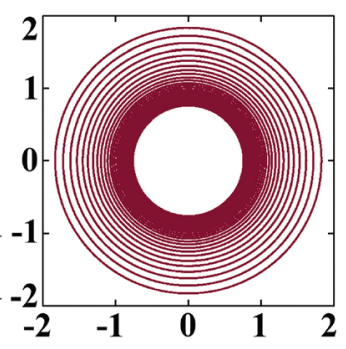

Figure 2. Regularities in band spacing. (a) Radii of two consecutive rings, $r_{n+1}$ vs $r_{n}$. The linearity of the plot confirms that the precipitation patterns obey the same spacing law as in ionic LR systems: ${ }^{18} r_{n+1} / r_{n}=p$, where $p$ is the spacing coefficient (in the plot, $p$ corresponds to the slope of the line). The specific data shown here are for the AgTMA/AgMUA system from Figure 1c, and the spacing coefficient is $p=1.02$. (b) The spacing coefficient decreases with increasing concentrations of either the outer or inner electrolyte (as predicted by the Matalon-Packter law ${ }^{13}$ ). The data shown in the graph are for two different concentrations (10.4 and $31.3 \mathrm{mM})$ of the AuTMA outer electrolyte and three different concentrations (1.13, 2.26, and $4.52 \mathrm{mM}$ ) of the AuMUA inner electrolyte. (c, d) Experimental (top row) and corresponding modeled (bottom row) images of two representative ring patterns for (c) the $10.4 \mathrm{mM}$ AuTMA/1.13 mM AuMUA system and (d) the $10.4 \mathrm{mM}$ AuTMA/2.26 mM AuMUA system.

While the analogies to the ionic Liesegang-type systems are manifest, the NP-based rings form according to a fundamentally different mechanism governed not by the solubility product of the salts but rather by the "threshold" NP aggregation at the point of NP electroneutrality (cf. Figure 1a). As we have previously shown, ${ }^{15,17}$ mixtures of oppositely charged NPs are stable in aqueous solutions if particles of either type are present in excess (these excess NPs form like-charged shells that stabilize small particle clusters ${ }^{19}$ ). However, when the charges on the NPs are balanced, the NPs rapidly aggregate and form large clusters whose diameters are on the order of $100 \mathrm{~nm} .{ }^{17}$ Moreover, if the concentration of NP clusters is above a certain threshold, $C^{*}(\sim 33$ $\mathrm{nmol}$ of clusters/mL or $\sim 1.1 \mathrm{mM}$ in terms of metal atoms), the clusters coalesce and precipitate from solution. ${ }^{15,17}$ In the case of our LR system, where concentrations vary in space and time, the condition for NP electroneutrality and cluster formation can be written as $Q^{+} c^{+}(x, t)=Q^{-} c^{-}(x, t)$, where the $Q^{\prime}$ 's are the NP charges and $c^{+}$and $c^{-}$are the concentrations of the positively and negatively charged NPs, respectively. Since the diffusive time scale in our systems (on the order of hours to days), is much larger than that for NP aggregation (seconds), the aggregation process in the regions where the electroneutrality condition is fulfilled can be treated as very rapid and proceeding until all of the NPs are aggregated. Mathematically, one can describe the formation of NP clusters behind the diffusion front by rates $R^{+}=\kappa c^{+}$and $R^{-}=\kappa c^{-}$, where the rate constant $\kappa$ is chosen to reflect the fact that aggregation is much faster than diffusion (i.e., the so-called Damköhler number, $D a$, is large; for simplicity, we chose $\kappa=1.0 \mathrm{~s}^{-1}$, for which $D a \approx$ $\left.10^{3}\right)$. In addition, the rate of coalescence/precipitation of the aggregated clusters can be written as $R_{\text {prec }}=k_{1} \hat{c} \Theta\left(\hat{c}-C^{*}\right)+k_{2} \hat{c} P$, where $\hat{c}$ is the concentration of the NP clusters, $P$ is the concentration of precipitate, $k_{1}$ and $k_{2}$ are rate constants (again, $k_{1}$ $=1.0 \mathrm{~s}^{-1}, k_{2}=1.0 \mathrm{~s}^{-1} \mathrm{M}^{-1}$, corresponding to $D a \approx 10^{3}$ ), and $\Theta$ is the Heaviside step function. This rate equation is similar to the one used in the models of ionic LR systems ${ }^{20}$ and accounts for (i) precipitation of clusters that commences when their local concentration reaches a threshold concentration ( $\hat{c} \geq C^{*}$; first term) and (ii) growth of precipitate already present at a given location by addition of neighboring clusters (even if $\hat{c}<C^{*}$; second term).

When this kinetics is combined with the diffusion of free NPs and the clusters they form (but not of the immobile precipitate), the process of pattern formation can be described by the so-called nucleation and growth model ${ }^{21}$ [for more details, see the Supporting Information (SI)] with mobile intermediate species (here, the clusters), for which the reaction-diffusion (RD) equations are of the form:

$$
\begin{gathered}
\frac{\partial c^{+}}{\partial t}=D^{+} \nabla^{2} c^{+}-R^{+} \\
\frac{\partial c^{-}}{\partial t}=D^{-} \nabla^{2} c^{-}-R^{-} \\
\frac{\partial \hat{c}}{\partial t}=D \nabla^{2} \hat{c}+R^{+}+R^{-}-R_{\mathrm{prec}} \\
\frac{\partial P}{\partial t}=R_{\mathrm{prec}}
\end{gathered}
$$

where $D^{+}=4.46 \times 10^{-11} \mathrm{~m}^{2} \mathrm{~s}^{-1}$ and $D^{-}=3.81 \times 10^{-11} \mathrm{~m}^{2} \mathrm{~s}^{-1}$ are the diffusion coefficients (measured using a Zetasizer NanoZS instrument, Malvern Instruments) of the positively and negatively charged NPs in the gel matrix, respectively. The diffusion coefficient of the clusters, $D \approx 5 \times 10^{-12} \mathrm{~m}^{2} \mathrm{~s}^{-1}$, was estimated from the Einstein-Stokes relation (on the basis of the average cluster size of $\sim 100 \mathrm{~nm}$ and with reference to $D^{+}$or $D^{-}$for the individual NPs). The boundary conditions are no flux at the boundaries of the gel, and the initial conditions at the junction between the two electrolytes are $c^{+}(r, t=0)=c_{0}^{+} \Theta\left(r_{0}-r\right)$ and $c^{-}(r, t=0)=c_{0}^{-} \Theta\left(r-r_{0}\right)$. The RD equations were solved using the "method of lines" technique with finite-difference discretization on a 1D (reflecting the system symmetry) equidistant grid followed by the integration of ordinary differential equations using the second-order Runge-Kutta method.

When implemented using experimental parameters, the model reproduced the experimentally observed ring-spacing trends (cf. Figures 2 and 3), though not the finite thicknesses of the bands. Importantly, the model suggests how the patterns of different morphological characteristics can be designed rationally by adjusting the relative NP charges. This is so because the changes in these charges affect the electroneutrality condition through the relation $Q^{-} / Q^{+}=c^{+}(x, t) / c^{-}(x, t)$ and therefore modify the spatial distribution of the NP clusters in the wake of the diffusion front. In general, decreasing the magnitude of the charge ratio increases the spacing coefficient. These effects are vividly illustrated in Figure 3 , which compares the rings formed in a system of AuMUA/ AgTMA NPs $\left(Q^{-} / Q^{+}=-1\right.$; Figure 3a) and in an NP pair in which the charge of the AuMUA NPs was decreased to $50 \%$ by "diluting" the SAM coating one type of NPs with neutral MUO $\left(Q^{-} / Q^{+}=\right.$ -0.5 ; Figure $3 b$ ). The spacing coefficient is 1.39 in the first system and increases to 1.53 in the second one. This result is in qualitative agreement with the predictions of the theoretical model (Figure 3c). 
a)
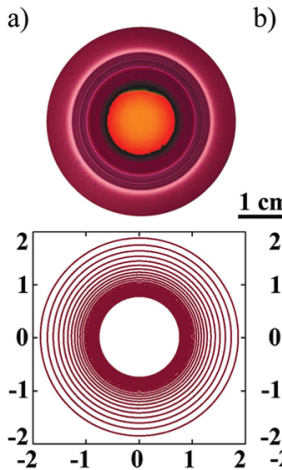

b)
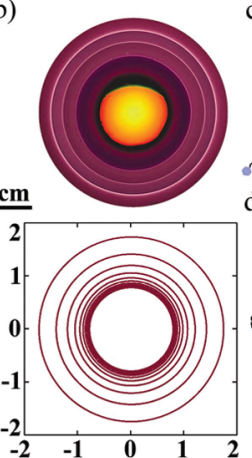

c)

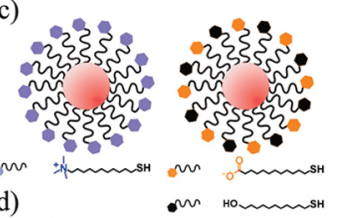

d)

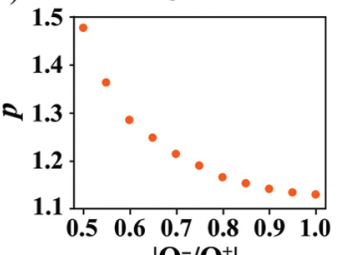

$\left|\mathbf{Q}^{-} / \mathbf{Q}^{+}\right|$

Figure 3. Effects of NP charges on pattern formation. (a, b) Experimental images of two systems differing in the ratios of NP charges: (a) AgTMA $(7.41 \mathrm{mM}) /$ AuMUA $(1.79 \mathrm{mM})$ pair characterized by $Q^{-} / Q^{+}=-1$, spacing coefficient $p=1.39$; (b) AgTMA (7.41 mM)/AuMUA/MUO (1.79 mM) pair characterized by $Q^{-} / Q^{+}=-0.5$ and $p=1.53$. The bottom row shows the corresponding simulated patterns. (c) Schematic illustration showing NPs coated with (left) a SAM of TMA and (right) a mixed SAM of MUA and MUO thiols. (d) Graph showing the theoretical dependence of $p$ on the absolute value of $Q^{-} / Q^{+}$. The spacing coefficient is predicted to increase with decreasing $\left|Q^{-} / Q^{+}\right|$, in qualitative agreement with experiment.

In contrast to traditional pattern-forming systems, in which all of the participating ions/molecules of a given type have identical sizes, the NPs are inherently polydisperse. An interesting consequence of NP polydispersity is illustrated in Figure S1a,b in the SI, where NPs of one type are significantly more polydisperse than the other (but the two types have the same average diameter). For example, when more polydisperse NPs are delivered into a gel containing less polydisperse NPs (Figure S1a), the first ring collects the largest and most polydisperse particles. As these larger particles of the outer electrolyte are effectively "filtered out," the consecutive rings are made of smaller and increasingly monodisperse NPs. In the opposite case (i.e., monodisperse NPs delivered into a gel containing polydisperse NPs; Figure S1b), the first ring contains the smaller, least polydisperse particles. While this result cannot be explained by the diffusion of the outer-electrolyte NPs, we hypothesize that the first ring collects preferentially the smaller, faster-diffusing particles of the inner electrolyte migrating from the gel's bulk toward the "incoming" front of the outer electrolyte. These smaller NPs reach the first precipitation zone before the larger ones, and the latter are "left behind" in the gel to form consecutive rings.

Finally, the phenomenon of NP-based ring formation can be extended to microscopic dimensions. This can be done, for example, using the so-called wet stamping (WETS) technique, ${ }^{6,22}$ in which an agarose stamp presenting an array of raised millimeter-sized features delivers the NPs of the outer electrolyte. Figure S1c shows two examples of patterns formed in this way, one originating from an array of stamped squares (left) and the other from an array of concave triangles (right). While the precipitation bands that form are thin $(50-100 \mu \mathrm{m})$, the method can currently produce only up to three rings, likely because of the small flux of NPs from the stamp into the gel film.

In summary, charged, "nanoionic" particles give rise to selforganization of Liesegang rings. Because the aggregation phenomena in this system are governed by the relative charges of the

organic SAMs on the surfaces of the NPs and not by the material properties of the particle cores, rings of different elemental compositions can be prepared. In addition, the ability to adjust the compositions of mixed SAMs on particle surfaces allows for variation of the spacing coefficient characterizing the ring patterns. In the future, it would be interesting to extend the phenomena we have described to other types of charged nanoobjects (notably DNA or charged proteins, which could be fractionated by the ring patterns) and also to test appropriately functionalized nanoparticles as "building blocks" for different pattern-forming systems, including those involving temporal oscillations and/or propagation of chemical waves.

Acknowledgment. This work was supported by an NSF Career Award (CTS-0547633, to B.A.G.) and an Alfred P. Sloan Fellowship (to B.A.G.).

Supporting Information Available: Additional experimental images and description of the model. This material is available free of charge via the Internet at http://pubs.acs.org.

\section{References}

(1) Henisch, H. K. Crystals in Gels and Liesegang Rings; Cambridge University Press: Cambridge, U.K., 1988

(2) Müller, S. C.; Ross, J. J. Phys. Chem. A 2003, 107, 7997.

(3) (a) Short, M. B.; Baygents, J. C.; Beck, J. W.; Stone, D. A.; Toomey, R. S.; Goldstein, R. E. Phys. Rev. Lett. 2005, 94, 18501. (b) Krug, H. J.; Brandtstadter, H.; Jacob, K. H. Geol. Rundsch. 1996, 85, 19. (c) Heaney, P. J.; Davis, A. M. Science 1995, 269, 1562.

(4) (a) Dolmetsch, R. E.; Xu, K. L.; Lewis, R. S. Nature 1998, 392, 933. (b) Falcke, M. Adv. Phys. 2004, 53, 255. (c) Hess, B. Naturwissenschaften 2000, 87, 199.

(5) Garfinkel, A.; Kim, Y. H.; Voroshilovsky, O.; Qu, Z. L.; Kil, J. R.; Lee, M. H.; Karaguezian, H. S.; Weiss, J. N.; Chen, P. S. Proc. Natl. Acad. Sci. U.S.A. 2000, 97, 6061

(6) Grzybowski, B. A. Chemistry in Motion: Reaction-Diffusion Systems for Micro- and Nanotechnology; Wiley: Chichester, U.K., 2009.

(7) (a) Liesegang, R. E. Naturwiss. Wochenschr. 1896, 11, 353. (b) Müller, S. C.; Kai, S.; Ross, J. Science 1982, 216, 635

(8) (a) Zhabotinsky, A. M.; Zaikin, A. N. Nature 1970, 225, 535. (b) Shinohara, S.; Seki, T.; Sakai, T.; Yoshida, R.; Takeoka, Y. Angew. Chem., Int. Ed. 2008, 47, 9039.

(9) Boyers, D. G.; Tiller, W. A. Appl. Phys. Lett. 1982, 41, 28

(10) (a) Sakurai, T.; Mihaliuk, E.; Chirila, F.; Showalter, K. Science 2002, 296, 2009. (b) Orbán, M.; De Kepper, P.; Epstein, I. R. J. Phys. Chem. 1982, 86,431 .

(11) Horváth, J.; Szalai, I.; De Kepper, P. Science 2009, 324, 772.

(12) (a) Al-Ghoul, M.; Sultan, R. J. Phys. Chem A 2003, 107, 1095. (b) Bena, I.; Droz, M.; Rácz, Z. J. Chem. Phys. 2005, 122, 204502. (c) Bena, I.; Droz, M.; Lagzi, I.; Martens, K.; Rácz, Z.; Volford, A. Phys. Rev. Lett. 2008, 101, 075701.

(13) (a) Matalon, R.; Packter, A. J. Colloid Sci. 1955, 10, 46. (b) Antal, T.; Bena, I.; Droz, M.; Martens, K.; Rácz, Z. Phys. Rev. E 2007, 76, 046203.

(14) Müller, S. C.; Kai, S.; Ross, J. J. Phys. Chem. 1982, 86, 4078.

(15) (a) Kalsin, A. M.; Kowalczyk, B.; Smoukov, S. K.; Klajn, R.; Grzybowski, B. A. J. Am. Chem. Soc. 2006, 128, 15046. (b) Kalsin, A. M.; Kowalczyk, B.; Wesson, P.; Paszewski, M.; Grzybowski, B. A. J. Am. Chem. Soc. 2007, 129,6664

(16) (a) Witt, D.; Klajn, R.; Barski, P.; Grzybowski, B. A. Curr. Org. Chem. 2004, 8, 1763. (b) Love, J. C.; Estroff, L. A.; Kriebel, J. K.; Nuzzo, R. G.; Whitesides, G. M. Chem. Rev. 2005, 105, 1103.

(17) Bishop, K. J. M.; Grzybowski, B. A. ChemPhysChem 2007, 8, 2171.

(18) Jablczynski, K. Bull. Soc. Chim. Fr. 1923, 33, 1592.

(19) Kalsin, A. M.; Grzybowski, B. A. Nano Lett. 2007, 7, 1018.

(20) Antal, T.; Droz, M.; Magnin, J.; Rácz, Z.; Zrínyi, M. J. Chem. Phys. 1998, 109,9479

(21) Bensemann, I. T.; Fialkowski, M.; Grzybowski, B. A. J. Phys. Chem. B 2005, 109, 2774.

(22) (a) Grzybowski, B. A.; Bishop, K. J. M.; Campbell, C. J.; Fialkowski, M.; Smoukov, S. K. Soft Matter 2005, 1, 114. (b) Campbell, C. J.; Smoukov, S. K.; Bishop, K. J. M.; Grzybowski, B. A. Langmuir 2005, 21, 2637. (c) Smoukov, S. K.; Bitner, A.; Campbell, C. J.; Kandere-Grzybowska, K.; Grzybowski, B. A. J. Am. Chem. Soc. 2005, 127, 17803.

JA906890V 